\title{
A Novel Method to Evaluate the Effect of Slope Blasting under Impact Loading
}

\author{
Wenhai Zhou $\mathbb{D}^{1},{ }^{1}$ Zhenbao Li, ${ }^{1}$ Xin Wu, ${ }^{1}$ Shujiang Wang, ${ }^{1}$ Yaru Lv, ${ }^{1}$ Xiaoming Lou, \\ Chaofei $\mathrm{Du}^{3}$ and Rui Liang $\mathbb{D}^{1}$ \\ ${ }^{1}$ School of Petrochemical Technology, Lanzhou University of Technology, Lanzhou 730050, China \\ ${ }^{2}$ Institute for Explosive Technology, Fuzhou University, Fuzhou 350116, China \\ ${ }^{3}$ Faculty of Engineering, China University of Geosciences, Wuhan 430074, China
}

Correspondence should be addressed to Wenhai Zhou; 18394499554@139.com and Rui Liang; liangr@lut.edu.cn

Received 24 September 2019; Revised 8 January 2020; Accepted 31 January 2020; Published 15 April 2020

Academic Editor: Rafał Burdzik

Copyright ( $(2020$ Wenhai Zhou et al. This is an open access article distributed under the Creative Commons Attribution License, which permits unrestricted use, distribution, and reproduction in any medium, provided the original work is properly cited.

In this paper, the quantitative evaluation of the explosion effect based on the fuzzy comprehensive evaluation method is proposed to describe the qualitative evaluation results. The selected state characteristic parameters are expressed by two kinds of membership functions, fuzzy normal and triangular distribution membership functions, and preliminary evaluation results are obtained. The validity index of the maximum membership principle is used to assess the accuracy of the evaluation results of two algorithms, and a relevant approaching degree is chosen to optimize the results. The entire evaluation process selects eleven indicators to form an evaluation set, including the boulder yield, root rate, flying distance of flyrock, explosive consumption, postcracking distance, detonator unit consumption, vibration velocity, loose coefficient, cast distance, throw rate, and blasted volume per meter of hole. Part of the indicator parameters are derived from field test monitoring, and another part of the indicator parameters are derived from numerical simulation. The simulation process uses the user-defined material interface function provided by LS-DYNA. And the numerical model of slope blasting is established by embedding the evolution relationship of tensile and compressive damage into the elastoplastic constitutive material. The evaluation method proposed in this paper is used to evaluate the postexplosion effect of Zijin Mountain gold-copper mine slope cast blasting. The results demonstrate that the fuzzy normal distribution membership function can correlate the state characteristic information and evaluation index effectively, and the working condition after explosion can be reflected accurately. Additionally, the influencing factors can be ranked by the importance degrees according to the calculated value of the evaluation index.

\section{Introduction}

Blasting technology is widely used in infrastructure construction, such as mine engineering, tunnel and underground engineering, municipal engineering, civil air defense and national defense engineering, water conservancy and hydropower engineering, and nuclear power construction engineering. In the last decade, most studies focus on optimization of blasting process parameters. Kuzu et al. [1, 2] highlighted that reasonable blasting design parameters and blasting effect can greatly improve production efficiency and achieve significant economic and social benefits. Wang et al. [3] identified the main parameters affecting the vibration spectrum based on a large number of explosion signals from a series of events to detect differences in continuous parameters. They also discussed how the parameters affect the occurrence and evolution of vibration signals. In recent researches, $\mathrm{Xu}$ et al. [4] investigated the dynamic response of deep rock masses during excavation of a stable blast. A new safety standard for blasting vibration of deep rock masses was proposed through field testing and numerical simulation and the failure mechanism of deep rock masses. Yan et al. [5] aimed to suggest a revised criterion to evaluate human response to the vibration from blasting excavation of a large-scale rock slope. Chen et al. [6] evaluated the stability of the slope by comprehensively analyzing the distribution of 
failure factors and vibration velocity characteristics. The parameters were analyzed by controlling the blasting load to optimize the blasting design. Albert et al. [7] measured results of seismic signals generated by airborne near-ground explosions of various types of explosives on the ground which showed that there are two different types of ground vibrations arriving, the earlier arrival time is mainly the underground path, and the explosion sound is close to the source. Through research and analysis, An et al. [8] concluded that there are more than 20 types of indices that affect rock-soil blasting and mainly focused on rock properties, explosive characteristics, and blasting parameters. However, because of designers' different understanding of the blasting principle and design method, different design results are often obtained, and the blasting effect is clearly different. The increasing maturity of computer technology and the improvement of the evaluation algorithm make it possible to solve these problems. By establishing a mathematical model, not only can the required blasting parameters be obtained but these parameters can also be optimized and the postblasting effect can be evaluated $[9,10]$. From the comparison of the development and application of BP network and fuzzy mathematics in risk prediction and assessment, Jong and Lee [11] earlier applied neural network (NN) models to training and testing models on the optimal NN model using collected data based on a series of observations and numerical experiments. The results showed that the neural network model can predict the powder coefficient. Xue [12] established a neural network model in which 14 geological parameters were selected as the network input, and the unit consumption of highway tunnel blasting explosives was calculated and the blasting vibration velocity was predicted. Ding et al. [13] developed digital three-dimensional (3D) processing software for cast blasting, which can be used to design the blasting process and prediction analysis of the postblasting effect. Dadashzadeh et al. [14] proposed an expert system to design presplit blasting in which parameters are calculated based on a three-layer BP (backpropagation) neural network. Additionally, Monjezi et al. [15], Li et al. [16], and Lei et al. [17] used gray correlation and a fuzzy mathematical algorithm to evaluate the blasting effect of step casting.

The postblasting effect of slope blasting is jointly determined by a number of evaluation indicators. The dimensions of each index are different, and the importance of the evaluation results is different. Therefore, the evaluation of postblasting effects is a vague problem. Fuzzy comprehensive evaluation is a method based on fuzzy mathematics, which measures factors with unclear boundaries and difficult to quantify. It can evaluate the membership status of the evaluated object from multiple factors. This method can reflect the importance of different evaluation indexes to the evaluation results through the setting of weights and can solve these types of problems similar to the postexplosion effect evaluation.

Based on the fuzzy comprehensive evaluation method for the step-cast blasting effect, in this paper, we mainly discuss the difference between the blasting effect evaluation results when membership functions for the factor evaluation set are selected for Gaussian and triangle distributions, and then relevant nearness analysis is chosen to optimize the results. We propose that when nonlinear factors, such as the impact of the blasting effect, are selected to measure the evaluation results of the target layer, the related membership function should be adopted to optimize the evaluation result so that the fuzzy probability obtained for the step-cast blasting effect can reflect engineering practice more accurately.

\section{Fuzzy Comprehensive Evaluation of Cast Blasting}

\subsection{Fundamentals}

(1) Establishing a set of factors for object of evaluation $U$ : for the subject of evaluation, the evaluation indicators are divided into multiple subsets $U=\left\{U_{1}\right.$, $\left.U_{2}, U_{3}, \ldots, U_{m}\right\}$, where $U_{i}$ is the factor that influences the object of evaluation.

(2) Establishing a set of evaluations $F$ : the evaluation set is a collection of the results for the object of evaluation, and $F=\left\{f_{1}, f_{2}, \ldots, f_{n}\right\}$ has $n$ evaluations of the state of each factor.

(3) Constructing an evaluation matrix: each factor of $U_{i}(i=1,2, \ldots, m)$ is evaluated to obtain the membership degree $r_{i j}$ of $U_{i}$ to $f_{j}(j=1,2, \ldots, n)$, and then the single factor evaluation set $r_{i}=\left(r_{i 1}, r_{i 2}\right.$, $\left.\ldots, r_{i n}\right)$ of $U_{i}$ is obtained. The evaluation matrix that consists of $m$ evaluated factors can be represented as follows:

$$
R=\left(r_{i j}\right)_{m \times n}=\left[\begin{array}{cccc}
r_{11} & r_{12} & \cdots & r_{1 n} \\
r_{21} & r_{22} & \cdots & r_{2 n} \\
\vdots & \vdots & \vdots & \vdots \\
r_{m 1} & r_{m 2} & \cdots & r_{m n}
\end{array}\right]
$$

(4) Comprehensive evaluation: the weights of each indicator are combined with the fuzzy relation matrix to determine the comprehensive evaluation set $D=$ $\left[d_{1}, d_{2}, \ldots, d_{n}\right]$, and the final evaluation result is determined by comparing $d_{1}, d_{2}, \ldots, d_{n}$ :

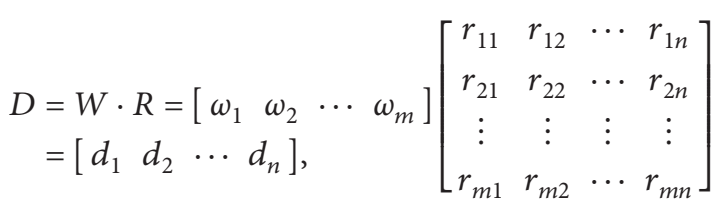

where $W, \omega_{i}$, and "." represent the comprehensive weight vector and the fuzzy operator, respectively. Different operator definitions have different fuzzy comprehensive evaluation models. The main factor determinant model is $M(\wedge, \vee)$, which corresponds to $d_{j}=V_{i=1}^{m}\left(\omega_{i} \wedge r_{i j}\right)$, and the weighted average model is $M(\cdot, \mathrm{V})$, which corresponds to $d_{j}=\min \left\{1, \sum_{i=1}^{m} \omega_{i} r_{i j}\right\}$. 
Typically, the main factor deterministic definition of the Zadeh operator is defined by the membership function, which is obtained by taking the larger, smaller, and remainder points in sequence. Therefore, in the specific process, the result of the operation often deviates from the nature of the problem because too much information is missing. It is better to use triangular modulus operators to reflect the nature of "And logic" and "Or logic." The influence of the main information, in addition to the impact of nonprimary information on the evaluation results, can be highlighted. Triangular modular operators include the drastic operator, Lukasiewicz operator, Einstein operator, Hamacher operator, Dubois-Prade operator, Yager operator, and Dombi operator, which are commonly used $[18,19]$. The Dombi operator is used in the main factor determinant $M(\wedge, \vee)$ to define the fuzzy operation, and the specific expressions of "And logic $\wedge$ " and "Or logic V" are represented as follows:

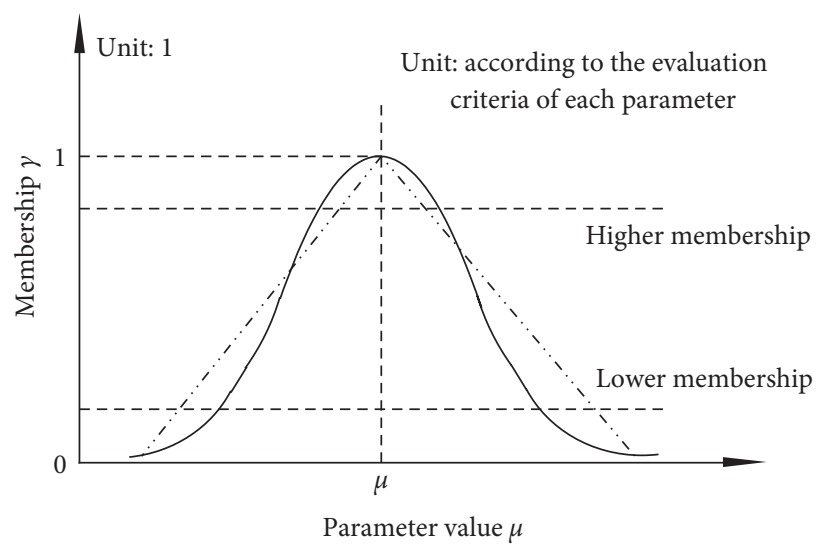

FIgURE 1: Comparison of membership in normal and triangular distributions.

$$
\begin{aligned}
& T_{\text {dop }}\left(\omega_{i}, r_{i j}\right)= \begin{cases}0, & \omega_{i}=0 \text { or } r_{i j}=0, \\
\frac{1}{1+\left[\left(\left(1 / \omega_{i}\right)-1\right)^{\xi}+\left(\left(1 / r_{i j}\right)-1\right)^{\xi}\right]^{1 / \xi},} & \text { others, }\end{cases} \\
& S_{\mathrm{dos}}\left(\omega_{i}, r_{i j}\right)= \begin{cases}0, & \omega_{i}=r_{i j}=0, \\
1, & r_{i j}=0 \text { or } \omega_{i}=0, \\
1+\frac{1}{\left[\left(\left(1 / \omega_{i}\right)-1\right)^{-\xi}+\left(\left(1 / r_{i j}\right)-1\right)^{-\xi}\right]^{-1 / \xi},} & \text { others, }\end{cases}
\end{aligned}
$$

where $\xi$ is a parameter, which requires $\xi>0$, and the value of $\xi=1$.

\subsection{Establishing the Membership Degree Function.} Typically, the form used to define the membership function can be divided into two types: quantitative and qualitative $[20,21]$. According to research and membership function principles, the Gaussian distribution quantitative method and triangle quantitative method have been used to study the effect of cast blasting separately. According to the nature of the selected factors, the factor set can be divided into two categories: smaller is better and larger is better.

A preliminary comparison of the Gaussian distribution and triangular function forms has demonstrated that when determining the interval of the parameter value, the Gaussian distribution function can better circumvent information with a low membership degree when the membership degree is small, and the Gaussian distribution function can better collect information with a high membership degree when the membership degree is large, as shown in Figure 1.

\subsubsection{Membership Degree Function of the Fuzzy Gaussian} Distribution. The evaluation units use a seminormal distribution near the extreme value when the Gaussian distribution function is selected for each parameter membership degree. The function can be expressed as follows:

$$
\gamma(u)=e^{-\left((u-\mu)^{2} / 2 \sigma^{2}\right)},
$$

where $\gamma(u)$ is the value of the membership degree of the evaluated element $u, \mu$ is the expected value, and $\sigma$ represents the width of the membership function.

Three comment sets are selected to evaluate the elements; the corresponding membership distribution functions are $\gamma_{\text {nor }}(u), \gamma_{\text {att }}(u)$, and $\gamma_{\text {abn }}(u)$; and the three interval distribution expectation values $\mu$ are in the ascending order: $\mu_{\min }, \mu_{0}$, and $\mu_{\max }$, where $\mu_{\min }$ and $\mu_{\max }$ are the minimum and maximum values of the evaluated elements and $\mu_{0}$ is the given expectation of the attention state (common). As is known from the characteristics of the Gaussian function, the area of $99.73 \%$ under the function curve is within the range of 3 standard deviations $(3 \sigma)$ around the expected value $\mu$. Therefore, $6 \sigma$ is 
chosen as the domain of the function in this paper, which is commonly used. In light of the randomness, fuzziness, and interval features of values of the geotechnical parameter, Zhang et al. [22] constructed the triangle and normal distribution membership functions of geotechnical parameters to evaluate the slope stability, respectively. It is concluded that the evaluation result is the best when the range of geotechnical parameters is $\pm 2.5 \sigma$ and $\pm 3 \sigma$, respectively. Juang et al. [23] and $\mathrm{Su}$ et al. [24] analyzed the reliability of slope stability when the geotechnical parameters are 1, 2, and 3 times the standard deviation. The results showed that the reliability is the best when 3 times the standard deviation is taken as the limit. The formulas are given as follows, where equations (6)-(8) are of the smaller-better type of the Gaussian function expression and equations (9)-(11) are of the larger-better type of the Gaussian function expression:

$$
\gamma_{\text {nor }}(u)=\left\{\begin{array}{ll}
1, & u \leq \mu_{\min }, \\
e^{-\left(\left(u-\mu_{\min }\right)^{2} / 2 \sigma_{\text {nor }}^{2}\right)}, & \mu_{\min }<u \leq \mu_{\max },
\end{array} \sigma_{\text {nor }}=\frac{\mu_{0}-\mu_{\min }}{3},\right.
$$

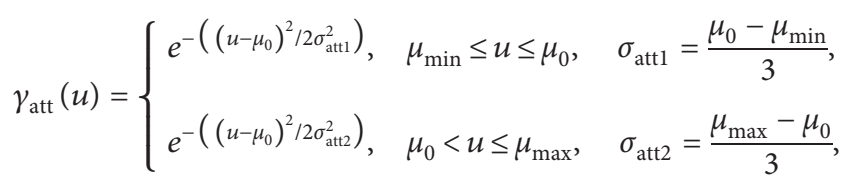

$$
\gamma_{\mathrm{abn}}(u)= \begin{cases}e^{-\left(\left(u_{\max }-\mu\right)^{2} / 2 \sigma_{\mathrm{abn}}^{2}\right)}, & \mu_{\min } \leq u<\mu_{\max }, \sigma_{\mathrm{abn}}=\frac{\mu_{\max }-\mu_{0}}{3}, \\ 1, & \mu \geq \mu_{\max },\end{cases}
$$

$$
\gamma_{\text {nor }}(u)=\left\{\begin{array}{ll}
0, & u \leq \mu_{\min }, \\
e^{-\left(\left(u-\mu_{\min }\right)^{2} / 2 \sigma_{\mathrm{nor}}^{2}\right)}, & \mu_{\min }<u \leq \mu_{\max },
\end{array} \sigma_{\text {nor }}=\frac{\mu_{0}-\mu_{\min }}{3},\right.
$$

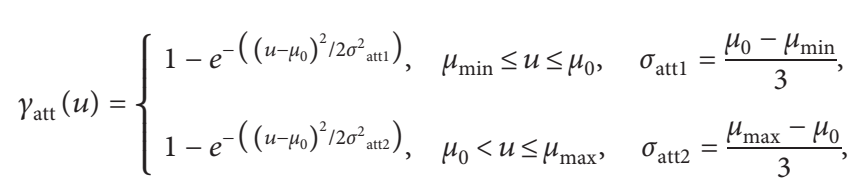

$$
\gamma_{\mathrm{abn}}(u)= \begin{cases}1-e^{-\left(\left(u_{\max }-\mu\right)^{2} / 2 \sigma_{a b n}^{2}\right)}, & \mu_{\min } \leq u<\mu_{\max }, \sigma_{\mathrm{abn}}=\frac{\mu_{\max }-\mu_{0}}{3} . \\ 1, & \mu \geq \mu_{\max },\end{cases}
$$

According to equations (6)-(11), the calculation model of each parameter for the three categories of membership degrees is determined, as shown in Figure 2.

2.2.2. Fuzzy Triangle Membership Degree Function. The specific expressions of the membership functions of the triangle distribution are listed. According to the parameter expectation value and distribution law of each evaluation interval of the fuzzy normal distribution, the formulas for the smaller-better type are equations (12)-(14) and the formulas for the larger-better type are equations (15)-(17):

$$
\begin{aligned}
& r_{\text {nor }}(\mu)= \begin{cases}1, & \mu<\mu_{\min }, \\
\frac{\mu_{\max }-\mu}{\mu_{\max }-\mu_{\min }}, & \mu_{\min } \leq \mu \leq \mu_{\max }, \\
0, & \mu>\mu_{\max },\end{cases} \\
& r_{\mathrm{att}}(\mu)= \begin{cases}1, & \mu<\mu_{\min }, \\
\frac{\mu-\mu_{\min }}{\mu_{\max }-\mu_{\min }}, & \mu_{\min }<\mu<\mu_{\max }, \\
1-\frac{\mu-\mu_{\max }}{\mu_{\max }-\mu_{\min }}, & \mu>\mu_{\max },\end{cases}
\end{aligned}
$$

$$
r_{\mathrm{abn}}(\mu)= \begin{cases}0, & \mu<\mu_{\min }, \\ 0, & \mu_{\min }<\mu<\mu_{\max }, \\ \frac{\mu-\mu_{\max }}{\mu_{\max }-\mu_{\min }}, & \mu>\mu_{\max }\end{cases}
$$

$$
r_{\mathrm{nor}}(\mu)= \begin{cases}\frac{\mu_{\min }-\mu}{\mu_{\max }-\mu_{\min }}, & \mu<\mu_{\min }, \\ 0, & \mu_{\min }<\mu<\mu_{\max }, \\ 0, & x>\mu_{\max },\end{cases}
$$

$$
r_{\mathrm{att}}(\mu)= \begin{cases}\frac{\mu}{\mu_{\max }-\mu_{\min }}, & \mu<\mu_{\min }, \\ \frac{\mu_{\max }-\mu}{\mu_{\max }-\mu_{\min }}, & \mu_{\min }<\mu<\mu_{\max }, \\ 0, & \mu>\mu_{\max }\end{cases}
$$

$$
r_{\mathrm{abn}}(\mu)= \begin{cases}0, & \mu<\mu_{\min }, \\ \frac{\mu-\mu_{\min }}{\mu_{\max }-\mu_{\min }}, & \mu_{\min }<\mu<\mu_{\max }, \\ 1, & \mu>\mu_{\max } .\end{cases}
$$

According to equations (12)-(17), the calculation model of each parameter for the three categories of membership is determined, as shown in Figure 3.

The bench cast blasting effect includes many aspects, and it is difficult to take every factor into consideration. Considering the comprehensive evaluation index system of the step-blasting effect in previous research $[16,17,25]$, 11 factors are selected as the evaluation indicators in this paper. Then, based on the results of 16 effective blasting operations conducted in [25] and others from 2009 to 2010 in a particular open-pit mine, parameters $\mu_{i}$ of the evaluation factors of step blasting are determined. The distribution expectations for the three evaluation intervals are shown in Table 1. Among them, the boulder yield, root 


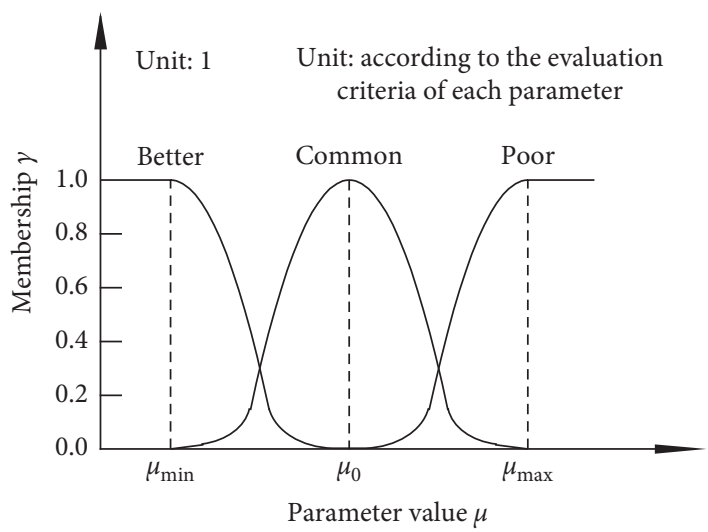

FIgURE 2: Model of membership in the fuzzy normal distribution.

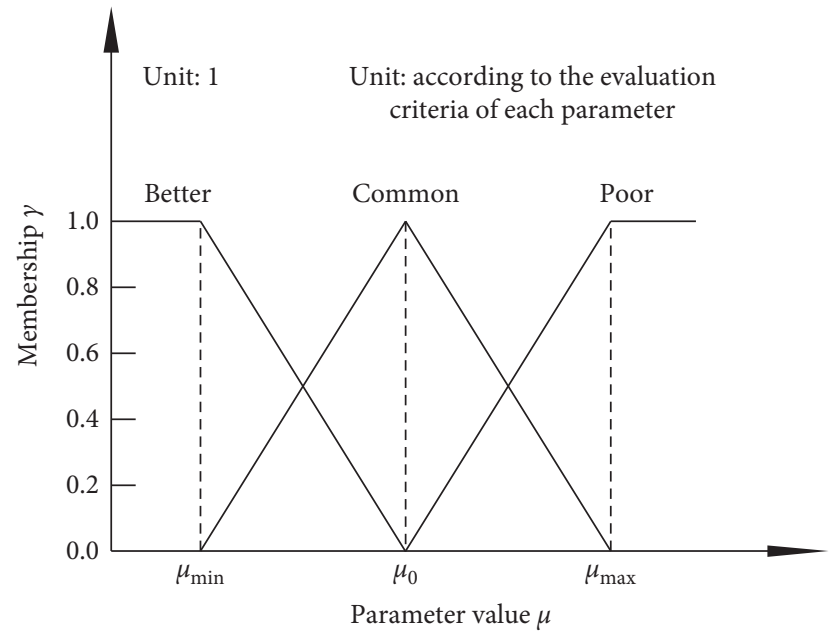

FIgURE 3: Model of membership in the triangular distribution.

rate, flying distance of flyrock, explosive consumption, postcracking distance, detonator unit consumption, and vibration velocity are of the smaller-better type. The four factors of the loose coefficient, casting distance, throw rate, and blasted volume per meter of hole are of the larger-better type. According to the expected value in Table 1, the membership calculation model of each parameter can be determined.

2.3. Index Weight Determination. The analytic hierarchy process is used to determine the weight.

(1) Establishing the evaluation matrix: using the expert survey method to analyze the importance of indicators in the state evaluation, the construction judgment matrix is as follows:

$$
A=\left[\begin{array}{cccc}
A_{11} & A_{12} & \cdots & A_{1 n} \\
A_{21} & A_{22} & \cdots & A_{2 n} \\
\vdots & \vdots & \cdots & \vdots \\
A_{m 1} & A_{m 2} & \cdots & A_{m n}
\end{array}\right],
$$

where $A_{i 12}$ is the importance degree of the evaluation index $i_{1}$ relative to $i_{2}$, and its value is from 1 to 9 .

(2) Calculating the weights of indicators: the weights of the index obtained by the analytic hierarchy process are as follows:

$$
\omega_{i}^{1}=\frac{\sqrt[m]{\prod_{i_{2}=1}^{m} A_{i_{1} i_{2}}}}{\sum_{i_{i}=1}^{m} \sqrt[m]{\prod_{i_{2}=1}^{m} A_{i_{1} i_{2}}}} .
$$

(3) Applying the consistency test to the judgment matrix: the matrix consistency ratio $C_{\mathrm{R}}$ is determined as follows:

$$
C_{R}=\frac{C_{I}}{R_{I}}=\frac{\left(\lambda_{\max }-m / m-1\right)}{R_{I}},
$$

where $\lambda_{\max }$ is the largest eigenvalue of the judgment matrix, $\lambda_{\max }=\sum_{i=1}^{m}\left(\left(A \omega_{i}^{1}\right) / n \omega_{i}^{1}\right)$, and $R_{I}$ is the average random consistency indicator. When $C_{R}<0.1$, the judgment matrix is considered to have acceptable consistency; otherwise, the judgment matrix needs to be readjusted. 
TABLE 1: Expected value of the state parameters.

\begin{tabular}{|c|c|c|c|c|c|}
\hline \multirow{2}{*}{ State parameter } & \multirow{2}{*}{ Unit } & \multirow{2}{*}{ Factor set } & \multicolumn{3}{|c|}{ Comment set } \\
\hline & & & $\operatorname{Better}\left(V_{1}\right), \mu_{\min }$ & Common $\left(V_{2}\right), \alpha$ & Poor $\left(V_{3}\right), \mu_{\max }$ \\
\hline Boulder yield & $\%$ & $u_{1}$ & 15 & 30 & 60 \\
\hline Root rate & $\%$ & $u_{2}$ & 0.1 & 0.4 & 0.8 \\
\hline Flying distance of flyrock & $\mathrm{m}$ & $u_{3}$ & 50 & 125 & 250 \\
\hline Explosive consumption & $\mathrm{kg} / \mathrm{m}^{3}$ & $u_{4}$ & 0.51 & 0.74 & 0.82 \\
\hline Postcracking distance & $\mathrm{m}$ & $u_{5}$ & 1.5 & 1.75 & 2.5 \\
\hline Detonator unit consumption & One $/ \mathrm{m}^{3}$ & $u_{6}$ & 0.01 & 0.04 & 0.1 \\
\hline Vibration velocity & $\mathrm{cm} / \mathrm{s}$ & $u_{7}$ & 0.5 & 1.25 & 2 \\
\hline & & & Poor $\left(V_{3}\right)$ & Common $\left(V_{2}\right)$ & $\operatorname{Better}\left(V_{1}\right)$ \\
\hline Loose coefficient & $\%$ & $u_{8}$ & 1.02 & 1.15 & 1.26 \\
\hline Cast distance & $\mathrm{m}$ & $u_{9}$ & 197 & 210 & 231 \\
\hline Throw rate & $\%$ & $u_{10}$ & 28.2 & 33 & 38.3 \\
\hline Blasted volume per meter of hole & $\mathrm{m}^{3} / \mathrm{m}$ & $u_{11}$ & 5 & 7.5 & 20 \\
\hline
\end{tabular}

\subsection{Effectiveness Index of the Maximum Subordination} Principle. In the fuzzy comprehensive evaluation, for the comment set $B=\left(b_{1}, b_{2}, \ldots, b_{n}\right)$, the relative index of the maximum membership principle of $\sum_{i=1}^{n} b_{i}=1$ is defined as

$$
\alpha=\frac{n \beta-1}{2 \gamma(n-1)},
$$

where $\beta=\max _{1 \leq i \leq n}\left\{b_{i}\right\}$ and $\gamma=\max _{1 \leq j \leq n, j \neq i}\left\{b_{j}\right\}$.

(1) When $\alpha=+\infty$, the maximum membership principle is fully effective.

(2) When $1 \leq \alpha<+\infty$, the maximum membership principle is very effective.

(3) When $0.5 \leq \alpha<1$, the maximum membership principle is slightly effective.

(4) When $0 \leq \alpha<0.5$, the maximum membership principle is the least effective.

(5) When $\alpha=0$, the maximum membership principle has no effect.

The validity of the $\alpha$ index enables the explanation of the relative confidence level of the implementation of the maximum membership principle and provides a quantitative description for the rationality of the evaluation results.

2.5. Approaching Degree Method. The approaching degree method is divided into symmetric closeness and asymmetric closeness. The former considers the comment level in the same level, whereas the latter considers the comment set in different positions. From the level of the blasting effect, the status of the commentary level is unequal, so in this paper, we choose the asymmetric sticking schedule method.

(1) Closeness $N\left(D_{j}, E_{j}\right)$ is the degree of closeness between the judgment object $D_{j}$ and its ideal subset $E_{i}(0,0$, $\ldots, 1,0, \ldots, 0)$. Standardize the evaluation object $D_{\mathrm{j}}$ series and rank it as $D_{j}, D_{j-1}, D_{j+1}, D_{j-2}, D_{j+2}, \ldots$. Convert $E_{\mathrm{i}}$ to $E_{1}\left(E_{1}=(1,0, \ldots, 0)\right)$ accordingly.

(2) Calculate the closeness as follows:

$$
\begin{array}{r}
N(D, E)=1-\frac{1}{n(n+1)} \sum_{k=1}^{n}\left|\mu_{D}^{P}\left(V_{k}\right)-\mu_{E}^{P}\left(V_{k}\right)\right| k \\
P=1,2 \cdots,
\end{array}
$$

where $\mu_{D}\left(V_{k}\right)$ is the membership of the object corresponding to $D$ belonging to $V_{k}$ and $P$ is a parameter that adjusts the calculation result. $P$ should be determined according to the specific scenario, and it is not easy to take a too large value. Otherwise, when there are more objects to be evaluated, the evaluation result will be detrimental to the grading. In this paper, $P=1\left(\mu_{D}\left(V_{k}\right)\right.$ is the membership of $V_{k}$ corresponding to $D$ ).

\section{Case Analysis}

3.1. Field Instance. The project of the cast blasting of a particular explosion area in the open pit of the Zijin Mountain gold-copper mine is selected as the evaluation object. The elevation of the slope is $544 \mathrm{~m}$ to $784 \mathrm{~m}$, and the relative height difference is $240 \mathrm{~m}$. The height of the explosion step is $12 \mathrm{~m}$, the width of the platform is $12 \mathrm{~m}$, the angle of the slope of the step is $75^{\circ}$, the hole spacing is $6.5 \mathrm{~m} \times 5 \mathrm{~m}$, and the unit consumption of explosives is controlled in the range of $0.61-0.65 \mathrm{~kg} / \mathrm{m}^{3}$. A blasting vibrometer is used in this construction, and the vibration test is conducted at a distance of approximately $150 \mathrm{~m}$ from the explosion area. The location of the explosion area, the effect after the explosion, and the arrangement of the vibrometer are shown in Figure 4.

The vibration wave velocity propagation law measured at the monitoring point is shown in Figure 5, and the vibrationrelated data measured are shown in Table 2. The three-axis direction is shown in Figure 6.

Through a field investigation and analysis after blasting, the results demonstrate that the block rate is approximately $42 \%$ and the root rate is approximately $0.25 \%$. The blasting rockfill looseness coefficient is approximately 1.2, and the farthest casting distance is up to $215 \mathrm{~m}$. According to Jiang et al. [26], 


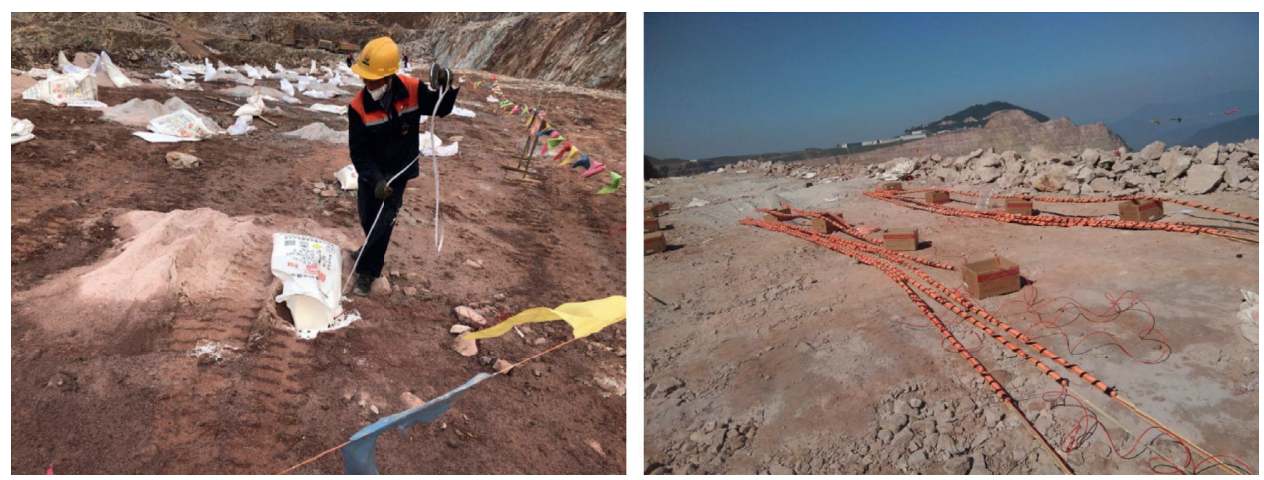

(a)
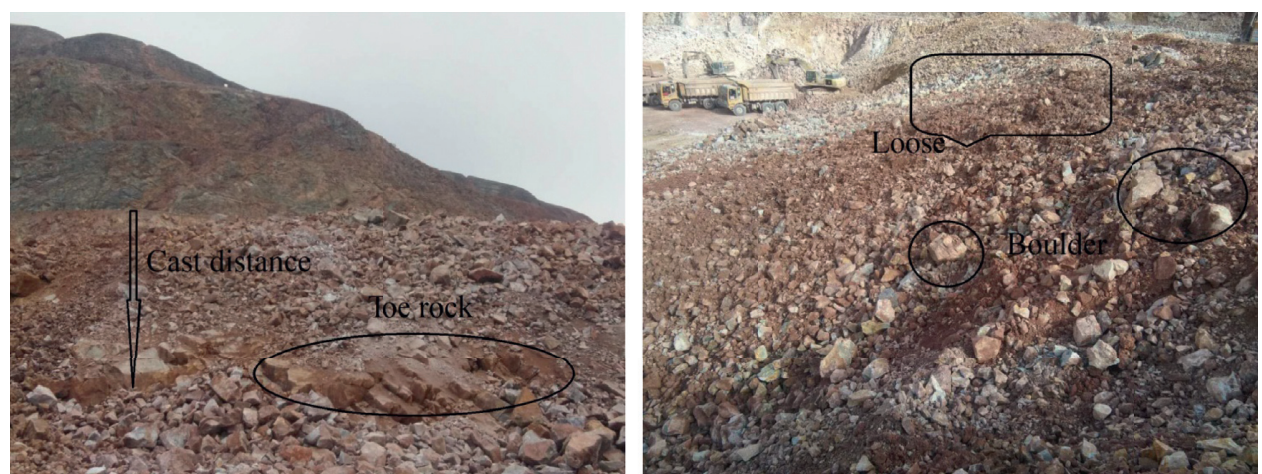

(b)

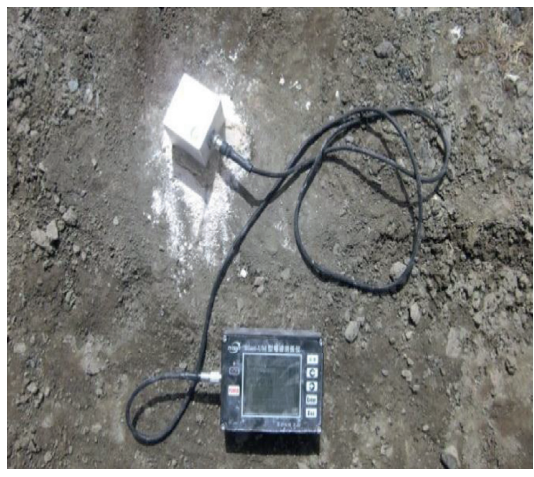

(c)

FIGURE 4: Explosion site and the effect after blasting. (a) Before the explosion. (b) After the explosion. (c) Mounting vibrometer.

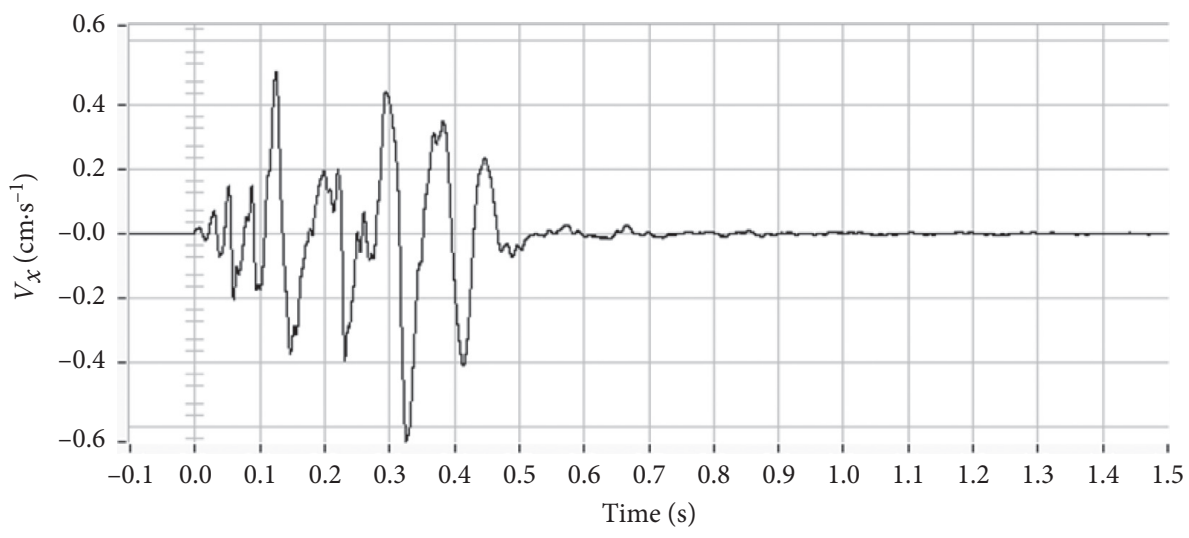

(a)

Figure 5: Continued. 


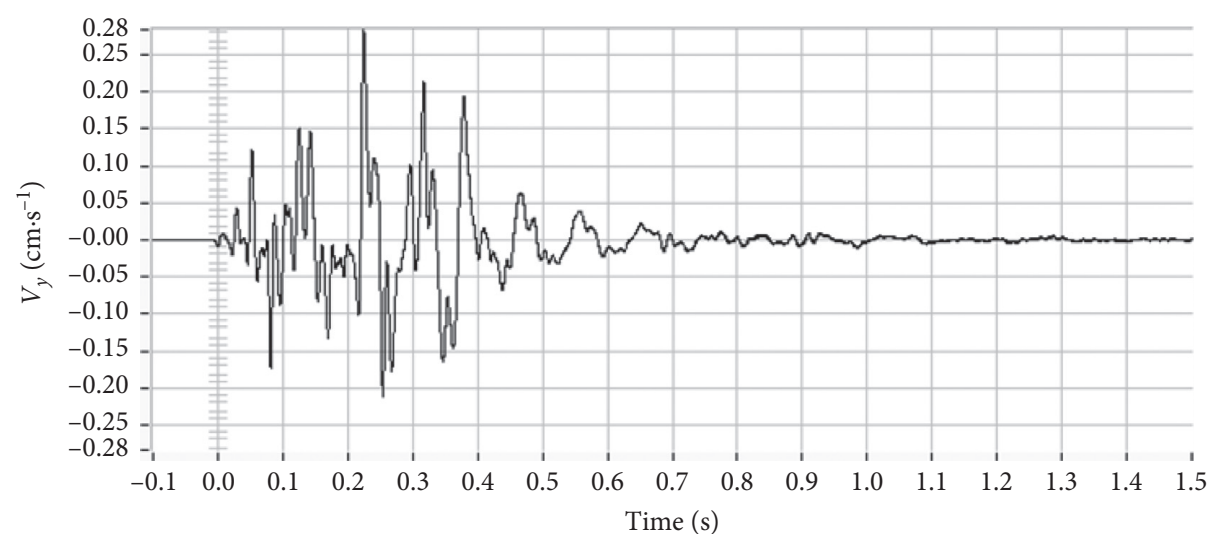

(b)

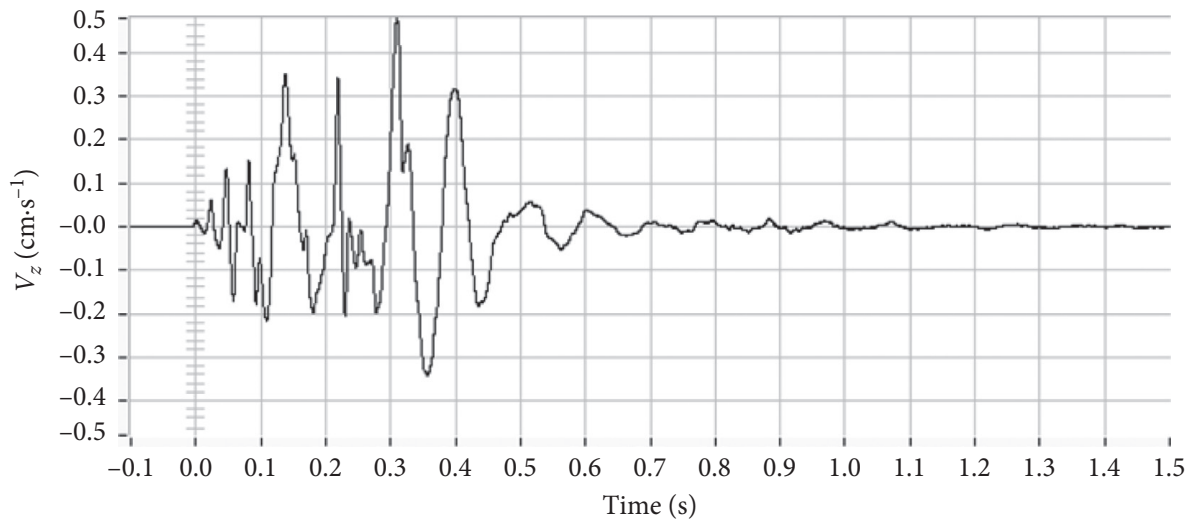

(c)

Figure 5: Vibration velocity of the monitoring point by the field test. (a) $X$-direction vibration of the monitoring point by the field test. (b) $Y$-direction vibration of the monitoring point by the field test. (c) $Z$-direction vibration of the monitoring point by the field test.

TABLE 2: Measured vibration-related data.

\begin{tabular}{lccccc}
\hline Channel name & Maximum $(\mathrm{cm} / \mathrm{s})$ & Half-wave frequency $(\mathrm{Hz})$ & FFT frequency $(\mathrm{Hz})$ & Range $(\mathrm{cm} / \mathrm{s})$ & Sensitivity coefficient $V(\mathrm{~m} / \mathrm{s})$ \\
\hline$X$-direction vibration & 1.1150 & 21.7 & 16.9 & 36.193 & 0.276 \\
$Y$-direction vibration & 1.2861 & 17.6 & 20.5 & 37.133 & 0.269 \\
$Z$-direction vibration & 0.4754 & 14.0 & 20.8 & 35.714 & 0.280 \\
\hline
\end{tabular}

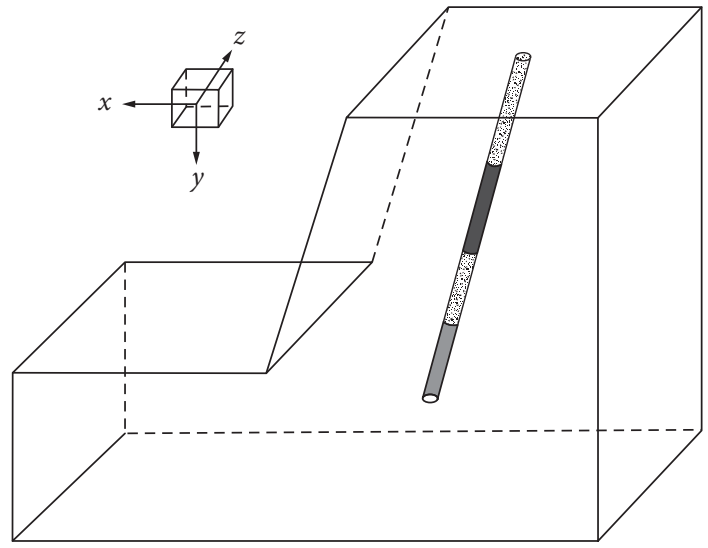

FIGURE 6: Illustration of the three-axis direction. 
TABle 3: Physical and mechanical parameters of rock.

\begin{tabular}{lccccccc}
\hline$\rho\left(\mathrm{g} / \mathrm{cm}^{3}\right)$ & $E(\mathrm{GPa})$ & $v$ & $\sigma_{0}(\mathrm{MPa})$ & $\beta$ & $G(\mathrm{GPa})$ & $K(\mathrm{GPa})$ & $K_{\mathrm{IC}}\left(10^{5} \mathrm{~N} \cdot \mathrm{m}^{-3 / 2}\right)$ \\
\hline 2.7 & 18.23 & 0.23 & 102 & 1.0 & 7.41 & 11.25 & 5.32 \\
\hline
\end{tabular}

Note: $\rho$ is the density, $E$ is Young's modulus, $v$ is Poisson's ratio, $\sigma_{0}$ is the yield stress, $G$ is the shear modulus, $K$ is the bulk modulus, $\beta$ is the enhancement factor, and $K_{\mathrm{IC}}$ is the fracture toughness of the medium.

TABLE 4: Parameters of the explosive.

\begin{tabular}{lcccccccc}
\hline$\rho\left(\mathrm{g} / \mathrm{cm}^{3}\right)$ & $p(\mathrm{GPa})$ & $A(\mathrm{GPa})$ & $B(\mathrm{GPa})$ & $R_{1}$ & $R_{2}$ & $\omega$ & $E_{0}(\mathrm{GPa})$ & $V_{0}$ \\
\hline 1.15 & 4.63 & 214 & 0.182 & 4.15 & 0.95 & 0.15 & 4.192 & 1.0 \\
\hline
\end{tabular}

Note: $A, B, R_{1}, R_{2}$, and $\omega$ are material parameters; $E_{0}$ is the initial specific energy; $p$ is the pressure; and $V_{0}$ is the initial specific volume of the explosive before detonation.

the ground particle vibration velocity is mainly determined by the $Y$-axis vibration velocity. Then, the vibration velocity of the blasting surface can be conservatively $1.1 \mathrm{~cm} / \mathrm{s}$.

\subsection{Simulation}

3.2.1. Setting Up Numerical Model according to Field Size. Based on the stage-cast blasting of an open pit in the Zijin Mountain gold-copper mine, the similarity 3D solid model of single hole detonation is established proportionally. Additionally, the values of the blasting process parameters and material parameters are in accordance with the actual scenario on-site. The overall specifications of the model are determined as follows by referring to the research experience by $\mathrm{Ma}$ et al. [27]: The measurements of the platform are a width of $12 \mathrm{~m}$, step slope of $75^{\circ}$, step height of $12 \mathrm{~m}$, and lower step height of $8 \mathrm{~m}$. To save computing time and space, and dynamically display the crack propagation and throwing tendency of the rock mass during blasting, the model thickness is defined as one unit thickness. For the rock material, a typical elastoplastic material is selected as the research object; that is, the MAT-PLASTIC-KINEMATIC model is adopted. The main physical and mechanical parameters of the rock are shown in Table 3, where $\rho$ is the density, $E$ is Young's modulus, $v$ is Poisson's ratio, $\sigma_{0}$ is the yield stress, $G$ is the shear modulus, $K$ is the bulk modulus, $\beta$ is the enhancement factor, and $K_{\mathrm{IC}}$ is the fracture toughness of the medium.

For the explosive, the 2\# rock emulsion explosive is selected, its material type is HIGH_EXPLOSIVE_BURN, and the state equation of explosives is selected without considering the JWL equation of the explosive product composition. The values of explosive material parameters are selected according to the simulation experience in $[28,29]$. The specific parameters are shown in Table 4, where $A, B, R_{1}$, $R_{2}$, and $\omega$ are material parameters; $E_{0}$ is the initial specific energy; $p$ is the pressure; and $V_{0}$ is the initial specific volume of the explosive before detonation.

The specific form of the JWL equation of the state when the explosive is exploded [28-30] is

$$
p=A\left(1-\frac{\omega}{R V}\right) e^{-R_{1} V}+B\left(1-\frac{\omega}{R V}\right) e^{-R_{2} V}+\frac{\omega E}{V} .
$$

\subsubsection{Constitutive Relation of Rock Mass Damage under Tension and Compression}

(1) Calculation Formula for Tensile Crack Damage. There are various kinds of cracks in the rock mass itself, also known as original damage, which further expands under the action of tensile stress of blasting load. Budiansky and O'connell [31] calculated the elastic modulus of the damage medium based on the "self-consistent method." The TCK model proposes that cracks in rock mass will be activated under the tensile stress of explosive load and the cracks will squeeze and collide with each other to form interaction force. The effective bulk modulus of a homogeneous rock mass with original damage can be expressed as

$$
\frac{K}{K_{0}}=1-\frac{16}{9} f_{1}(v) C_{\mathrm{d}}=1-D
$$

where $K$ and $K_{0}$, respectively, represent the bulk modulus of the damage medium and initial bulk modulus; $f_{1}(v)=\left(\left(1-v^{2}\right) /(1-2 v)\right)$, in which $v$ is Poisson's ratio; $C_{\mathrm{d}}$ is the medium crack density; and $D$ is the damage scalar.

Xie et al. [32] put forward Poisson's ratio change formula of the rock mass medium under impact load:

$$
v=v_{0}\left(1-\frac{16}{9} C_{\mathrm{d}}\right)
$$

where $v_{0}$ is the initial Poisson's ratio of the medium.

Grady and Kipp [33] proposed that the number of original damage cracks $N$ in a unit medium under impact tensile stress can be accurately calculated by Weibull function:

$$
N=\xi\left(\frac{p}{3 k}\right)^{m}
$$

where $p$ is the impact stress and $\xi$ and $m$ represent the distribution parameters of Weibull function. 
Taylor et al. [34] proposed a formula for the radius of fragments in brittle media such as rock mass under dynamic impact loading:

$$
r=\frac{1}{2}\left(\frac{\sqrt{20} K_{\mathrm{IC}}}{\rho C \dot{\varepsilon}_{v, \max }}\right)^{2 / 3}
$$

where $K_{\mathrm{IC}}$ and $\rho$, respectively, represent the fracture toughness and density of the medium, $C$ is the longitudinal wave velocity, and $\dot{\varepsilon}_{v \text {, max }}$ represents the volume strain rate at fracture. The functional relationship between the average radius of fragments $r$ and the crack density $C_{\mathrm{d}}$ can be expressed as

$$
C_{\mathrm{d}}=N \omega r^{3}
$$

where $\omega$ is the proportional coefficient.

Substituting formulas (26) and (27) into (28) gives

$$
C_{\mathrm{d}}=\xi\left(\frac{p}{3 k}\right)^{m}\left[\omega \cdot \frac{1}{8}\left(\frac{\sqrt{20} K_{\mathrm{IC}}}{\rho C \dot{\varepsilon}_{v, \max }}\right)^{2}\right]=\frac{5}{2} \xi \bowtie\left(\frac{p}{3 k}\right)^{m}\left(\frac{K_{\mathrm{IC}}}{\rho \mathrm{C} \dot{\varepsilon}_{v, \max }}\right)^{2}
$$

Taking $k=\xi \bowtie$ in equation (29) yields

$$
C_{\mathrm{d}}=\frac{5}{2} k\left(\frac{P}{3 K}\right)^{m}\left(\frac{K_{\mathrm{IC}}}{\rho C}\right)^{2} \dot{\varepsilon}_{v \max }^{-2}
$$

The formula of tensile crack damage function can be obtained from equation (24) as

$$
D=\frac{16}{9} f_{1}(\bar{v}) C_{\mathrm{d}}
$$

From the generalized Hook's law, the stress-strain function relationship is as follows:

$$
\Delta \sigma_{i j}=K_{d} \delta_{i j} \Delta \varepsilon_{v}+2 G_{d} \Delta e_{i j}
$$

where $K_{d}$ and $G_{d}$ represent the modulus of the damaged medium and $\Delta \varepsilon_{v}$ and $\Delta e_{i j}$ are body strain increments and partial strain increments, $\Delta \varepsilon_{v}=3 \varepsilon_{\mathrm{m}}$, in which $\varepsilon_{\mathrm{m}}$ is the average normal stress.

(2) Constitutive Relationship of Hardening Effect under Compressive Stress. Zhang et al. [35] pointed out in the report that the elastoplastic constitutive relationship of the rock mass medium obeying isotropic hardening under the action of detonation gas compression (yield equation) can be expressed as

$$
\Phi=\sigma_{i}^{2}-\sigma_{y}^{2}
$$

where $\sigma_{i}=\sqrt{(3 / 2) s_{i j}}$. According to the generalized Hooke's law, the tensor form is used to represent the deviatoric stress $S_{\mathrm{ij}}$ as

$$
s_{i j}=\sigma_{i j}-\frac{1}{3} \sigma_{k k} \delta_{i j}
$$

During the explosion process, the functional relationship between the effective plastic strain $\varepsilon_{\text {eff }}^{P}$ of the rock mass and the yield limit $\sigma_{y}$ of the fracture failure under the dynamic load is

$$
\begin{aligned}
& \varepsilon_{\mathrm{eff}}^{P}=\int_{0}^{t} \sqrt{\frac{3}{2}} \dot{\varepsilon}_{i j}^{P} \mathrm{~d} t, \\
& \sigma_{y}=\sigma_{0}+\beta E_{p} \varepsilon_{\mathrm{eff}}^{P},
\end{aligned}
$$

where $E_{p}$ is the plastic hardening modulus, usually set as $0.1 E, \beta$ is the hardening parameter, and $\dot{\varepsilon}_{i j}^{p}=\dot{\varepsilon}_{i j}-\dot{\varepsilon}_{i j}^{e}$. In formula (33), when $\Phi \leq 0$, the condition is elastic or neutral loading; when $\Phi>0$, the condition is plastic hardening.

(3) Pull-Pressure Damage Constitutive Model Embedding Simulation Software. The large-scale finite element analysis software LS-DYNA provides a rich material library for instantaneous power and large deformation damage simulation and also provides users with a convenient model import window. In this study, the calculation model of the cracking damage of the slope rock mass with cracks and the constitutive relation of the hardening effect under compressive stress are coupled; then the simulation software is imported for step-blasting calculation, which can simulate the trajectory of the throwing motion and the fragmentation of the rock mass under the impact of the explosion. The specific process is shown in Figure 7.

\subsection{Simulation Result Analysis}

3.3.1. Casting State of Motion. The distribution of the casting state of the rock at different times is shown in Figure 8. The model initiates the cast velocity from the foot of the slope at $7 \mathrm{~ms}$. As time elapses, the casting phenomenon evolves from the foot of the slope to the free surface. By monitoring the model casting units, we found that the maximum casting velocity appeared at the middle of the blast-hole pack, with a velocity of $21.6 \mathrm{~m} / \mathrm{s}$. Additionally, this value is consistent with the conclusion regarding the maximum casting velocity proposed by Huang et al. [36, 37], which is $18-28 \mathrm{~m} / \mathrm{s}$. The average scattering distance is approximately $47 \mathrm{~m}$, the postcracking distance is approximately $1.2 \mathrm{~m}$, and the casting rate is approximately $34 \%$.

3.3.2. Rock Mass Fragmentation Analysis. The bulks are mainly distributed at the foot of the slope, the sides of the contact surface between the explosive and the blockage, and the free surface at the top of the step. The bulks at the bottom of the slope mainly depend on the selection of the chassis resistance line value, and the difference in the resistance line value results in different bulk distributions. As shown for rock No. 5 in Figure 9, the diameter is approximately $2.3 \mathrm{~m}$. The bulks appear on both sides of the contact surface of the explosive, and the blockage is mainly caused by the different mechanical properties and physical parameters of the 


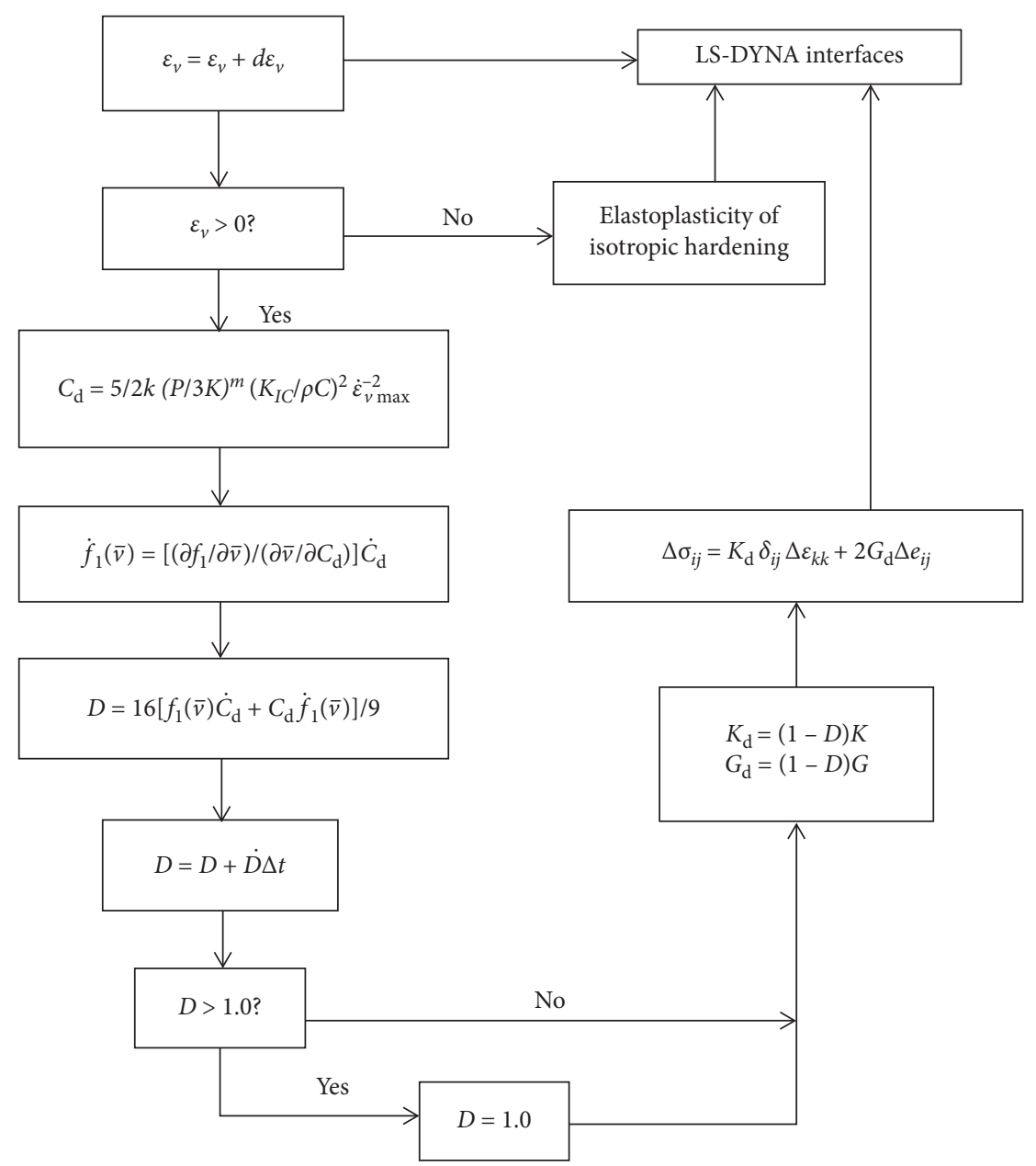

FIgURE 7: Flowchart of the damage model.

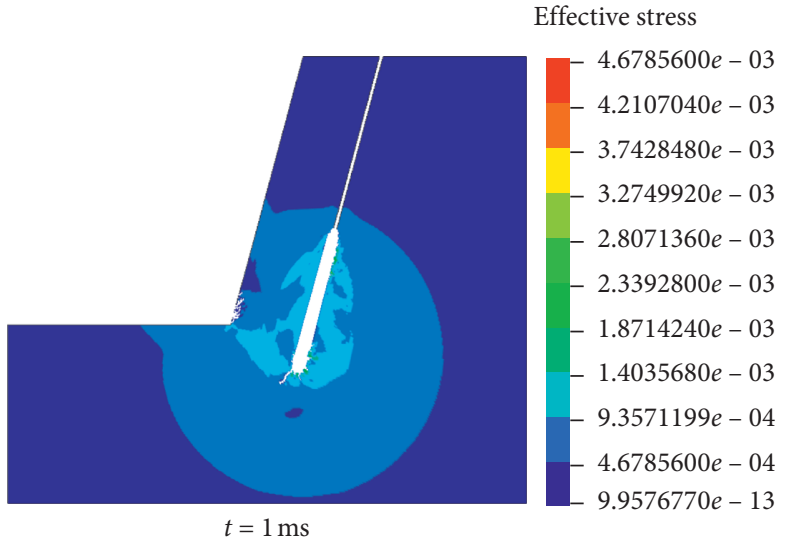

(a)

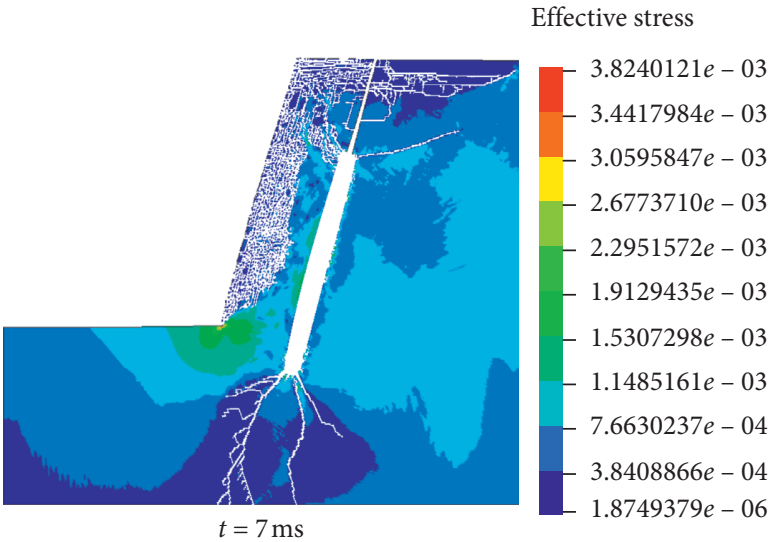

(b)

FIgUre 8: Continued. 


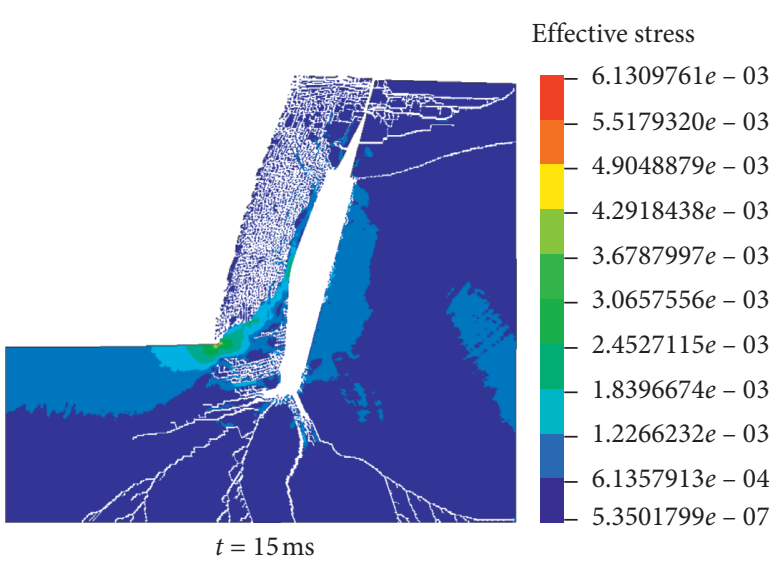

(c)

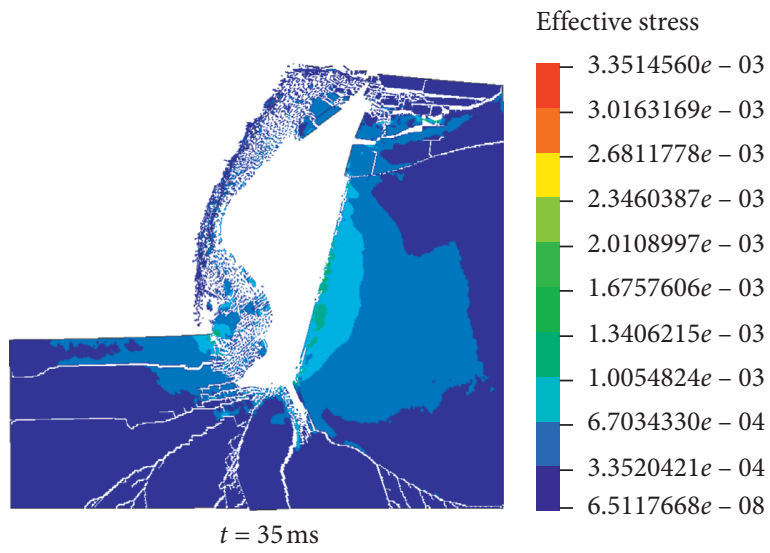

(e)

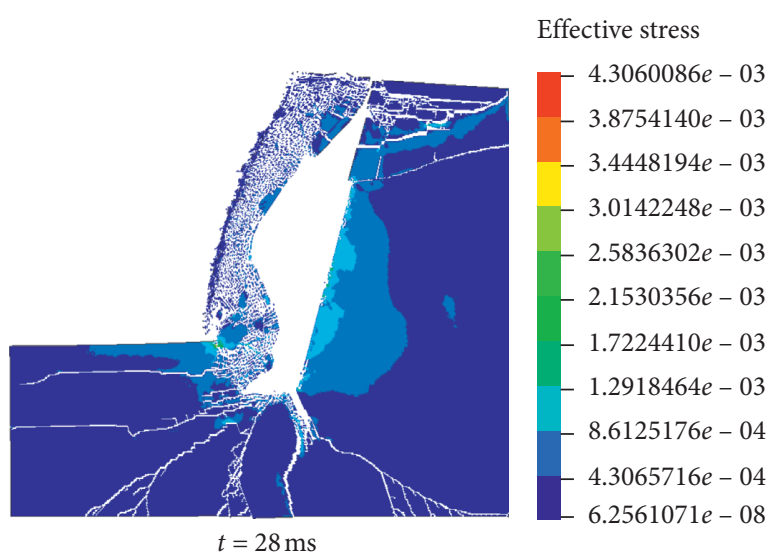

(d)

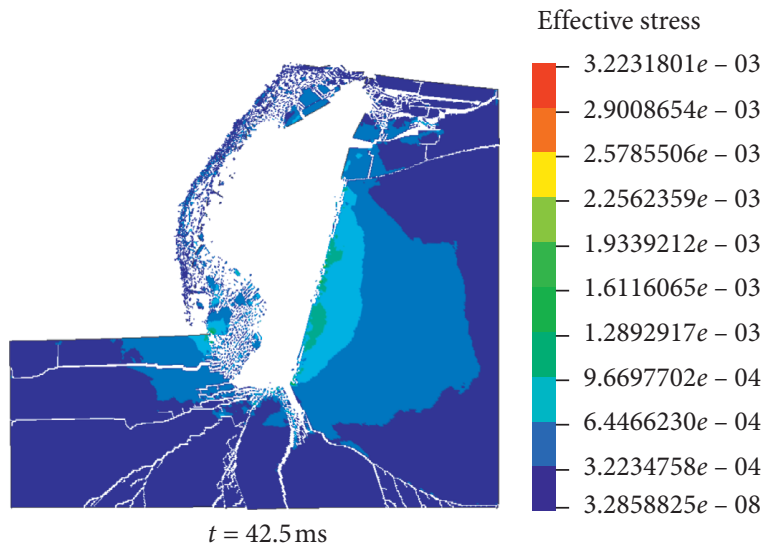

(f)

Figure 8: Variety of damage and deformation of the rock at different times.

material on both sides of the contact surface. The stress wave is reflected and transmitted at the contact surface, and the reflection stretch acts on the penetrating microcrack, which causes the rock mass to break away from the parent rock under tensile stress to form bulks. As shown in rock block Nos. 1 and 2 and Nos. 3 and 4 in Figure 9, the diameter distribution is in the range of 1.6 to $2.7 \mathrm{~m}$. The bulks appear on the free surface at the top of the step because of the transmission of stress waves at the interface of the explosive and blockage to the top free surface. The transmitted wave also forms a reflection stretching effect, which causes the rock mass in the region to break along the internal microfracture to form bulks. As shown in rock block No. 6 in Figure 9, the diameter is approximately $1.7 \mathrm{~m}$.

\subsubsection{Analysis of Simulated Vibration Wave Velocity. A} particle at the surface location of the simulation model is selected as the vibration speed-monitoring station, the installation position of the vibration-measuring instrument in the monitoring point and the field monitoring test is spatially consistent, and the specific change law is shown in Figure 10 . The simulated three-axis peak vibration velocity of the monitoring points is $V_{x}=0.68 \mathrm{~cm} / \mathrm{s}, V_{y}=0.74 \mathrm{~cm} / \mathrm{s}$, and $V_{z}=0.59 \mathrm{~cm} / \mathrm{s}$, respectively. The triaxial velocity variation law, the peak size, and the time point at which the peak occurs are in good agreement with the data obtained by the vibrometer monitoring, It is reasonable to take the surface vibration velocity $V_{y}=1.1 \mathrm{~cm} / \mathrm{s}$, when the effect evaluation is carried out in the text (the three-axis velocity direction is consistent with the data in Figure 6).

\subsection{Fuzzy Comprehensive Evaluation}

3.4.1. The Basic Steps. According to the actual postblasting scenario and a similar simulation test, the 11 evaluation indicators selected are considered sequentially, and the set of evaluation object factors is established as follows:

$U=\{42,0.25,47,0.63,1.2,0.07,1.1,1.2,215,34.1,6.3\}$.

Step 1 (determining the membership degree of each index). The factor set $U$ of the evaluation objects is substituted into equations (6)-(11), and the evaluation matrix determined by the Gaussian distribution membership function is obtained after calculation: 


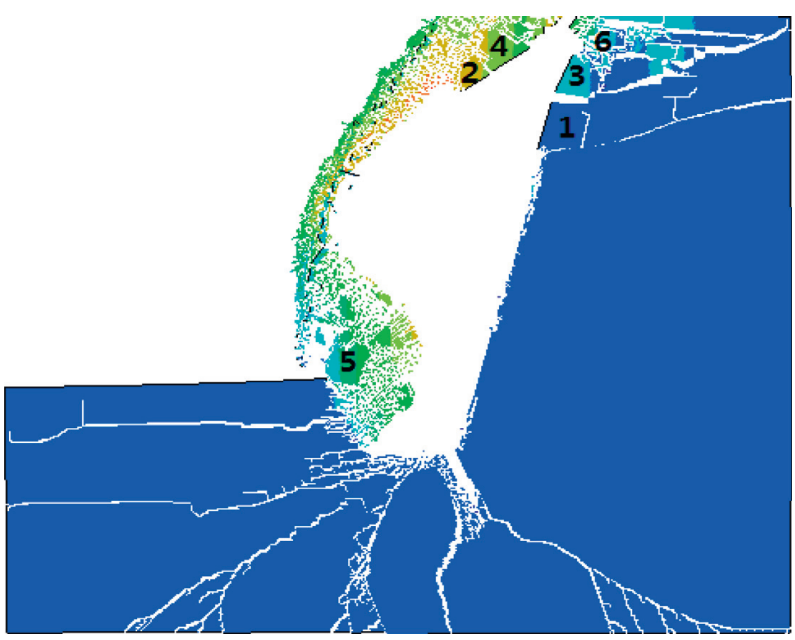

FIGURE 9: Distribution of rock blocks.

$$
R_{\mathrm{if} \text { (gaussian) }}=\left[\begin{array}{ccccccccccc}
0 & 0.325 & 1 & 0.294 & 1 & 0 & 0.056 & 0.738 & 0.926 & 0.946 & 0 \\
0.487 & 0.325 & 0 & 0.357 & 0 & 0.325 & 0.011 & 0.177 & 0.225 & 0.176 & 0.645 \\
0.198 & 0 & 0 & 0 & 0 & 0.325 & 0.197 & 0 & 0 & 0 & 0.355
\end{array}\right]^{T} .
$$

The factor set $U$ of the evaluation objects is substituted into equations (12)-(17), and the evaluation matrix determined by the triangle membership function is obtained after calculation:

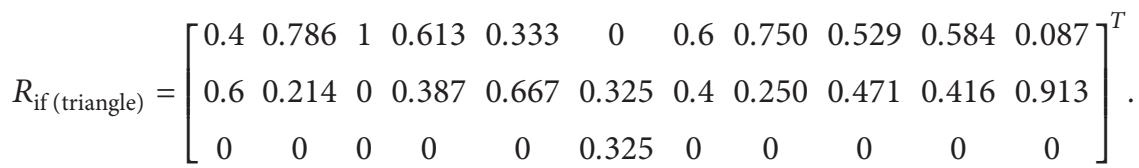

In the factor set, the smaller-better type of evaluation objects $u_{1}, u_{2}, u_{3}, u_{4}, u_{5}, u_{6}$, and $u_{7}$ is substituted into equations (6)-(8) and (12)-(14) for calculation, whereas the larger-better type $u_{8}, u_{9}$, $u_{10}$, and $u_{11}$ is substituted into equations (9)-(11) and (15)-(17) for calculation. The comment set constructed is of the decreasing type in this paper, so the evaluation object is calculated in a smaller-better membership function, and the calculated values are sequentially put into the evaluation matrix $R_{\text {if }}$ according to the order of comments. Conversely, the membership values calculated by substituting the evaluation object into a larger-better membership function need to be sorted back first and then put into the evaluation matrix $R_{\text {if }}$ in turn.

Step 2 (determining the weight of each index using the $A H P$ ). The judgment matrix is constructed as follows using the expert investigation method according to equation (18):

$$
R=\left[\begin{array}{lllllllllll}
1 & 5 & 6 & 1 & 7 & 7 & 3 & 4 & 6 & 3 & 2 \\
\frac{1}{5} & 1 & 1 & \frac{1}{5} & 3 & 2 & \frac{1}{3} & \frac{1}{2} & 1 & \frac{1}{3} & \frac{1}{4} \\
\frac{1}{6} & 1 & 1 & \frac{1}{6} & 2 & 1 & \frac{1}{4} & \frac{1}{3} & \frac{1}{2} & \frac{1}{5} & \frac{1}{5} \\
1 & 5 & 6 & 1 & 7 & 7 & 2 & 3 & 4 & 2 & 1 \\
\frac{1}{7} & \frac{1}{3} & \frac{1}{2} & \frac{1}{7} & 1 & 1 & \frac{1}{4} & \frac{1}{4} & \frac{1}{3} & \frac{1}{5} & \frac{1}{6} \\
\frac{1}{7} & \frac{1}{2} & 1 & \frac{1}{7} & 1 & 1 & \frac{1}{4} & \frac{1}{3} & \frac{1}{2} & \frac{1}{4} & \frac{1}{5} \\
\frac{1}{3} & 3 & 4 & \frac{1}{2} & 4 & 4 & 1 & 1 & 2 & 1 & \frac{1}{2} \\
\frac{1}{4} & 2 & 3 & \frac{1}{3} & 4 & 3 & 1 & 1 & 1 & \frac{1}{2} & \frac{1}{3} \\
\frac{1}{6} & 1 & 2 & \frac{1}{4} & 3 & 2 & \frac{1}{2} & 1 & 1 & \frac{1}{3} & \frac{1}{4} \\
\frac{1}{3} & 3 & 5 & \frac{1}{2} & 5 & 4 & 1 & 2 & 3 & 1 & 1 \\
\frac{1}{2} & 4 & 5 & 1 & 6 & 5 & 2 & 3 & 4 & 1 & 1
\end{array}\right]
$$




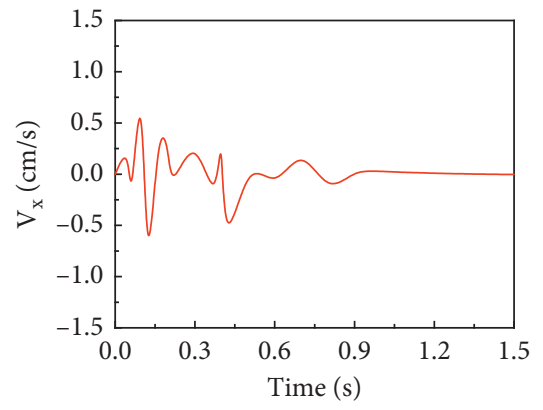

— X vibration velocity

(a)

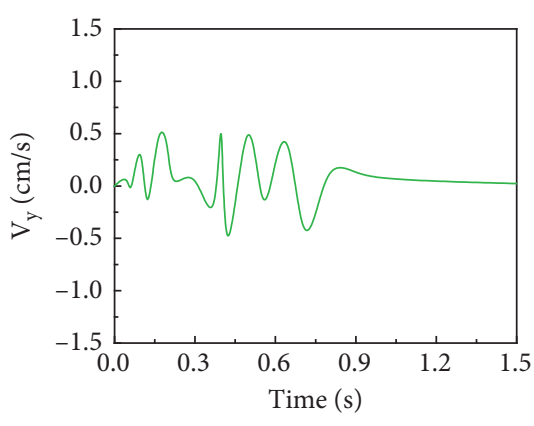

Y Y vibration velocity

(b)

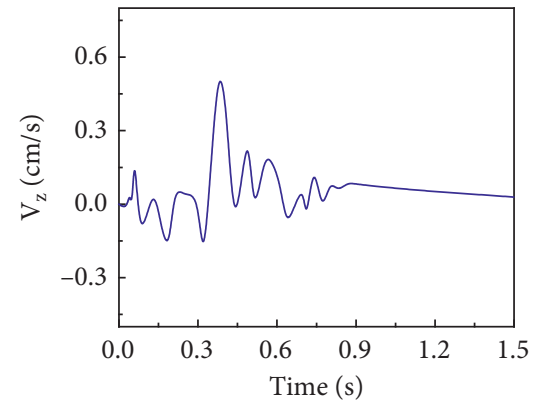

— Z vibration velocity

(c)

FIGURE 10: Vibration velocity of the monitoring point by numerical simulation. (a) $X$-direction vibration of the monitoring point by numerical simulation. (b) $Y$-direction vibration of the monitoring point by numerical simulation. (c) $Z$-direction vibration of the monitoring point by numerical simulation.

TABLE 5: Comparison of fuzzy comprehensive evaluations.

\begin{tabular}{|c|c|c|c|c|}
\hline \multirow{2}{*}{ Evaluation object } & \multirow{2}{*}{ Membership function distribution } & \multicolumn{3}{|c|}{ Membership degree } \\
\hline & & $\operatorname{Better}\left(V_{1}\right), b_{\text {nor }}$ & Common $\left(V_{2}\right), b_{\text {att }}$ & Poor $\left(V_{3}\right), r_{\mathrm{abn}}$ \\
\hline \multirow{2}{*}{ Blasting effect } & Gaussian distribution & 0.227 & 0.409 & 0.364 \\
\hline & Triangle distribution & 0.493 & 0.507 & 0 \\
\hline
\end{tabular}

The weight vector is obtained after normalizing each index according to equation (19):

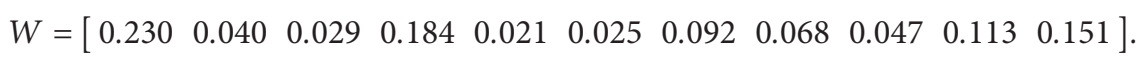

$C_{\mathrm{R}}$ is obtained after calculation according to the formula $C_{\mathrm{R}}=0.052<0.1$, which shows that the judgment matrix is consistent and there is no need to revise it.

Step 3 (comprehensive evaluation). The evaluation matrix $R$ obtained after calculation and weight vector $W$ obtained after normalization are substituted into equations (2)-(4) for calculation to obtain the evaluation results, which are presented in Table 5.

According to the maximum membership degree principle, the evaluation results of this blasting result controlled by the Gaussian distribution membership function and triangle membership function are both good. However, from the numerical distribution of each component of the two evaluation results, it is obvious that there are great differences between the two evaluation results, which show that the maximum membership principle is invalid in this case. This is because, in the application process, the maximum membership degree principle loses the information of nonmajor components in the evaluation results, which leads to the obvious unreasonable evaluation results in Table 5 .
3.4.2. Analysis of the Effectiveness Index of the Maximum Membership Degree Principle. The results obtained by the two methods of evaluation are substituted into equation (21) for calculation, and two effectiveness indices are obtained as follows: $\alpha_{\text {(gauss) }}=0.47$ and $\alpha_{\text {(triangle) }}=0.46$. The range of the confidence level judged according to the implementation of the principle of maximum membership indicates that the principle of maximum membership is inefficient in this case. Therefore, using the closeness analysis method is considered to process the evaluation results to determine the specific level of the evaluation object. The nearness method is considered to process evaluation results to determine the specific grade of the evaluation objects.

3.4.3. Applying the Nearness Method to Analyze the Evaluation Results. The results of two types of evaluation are input into equation (22) for calculation. Two evaluation result vectors controlled by the Gaussian distribution and triangle membership function are, respectively, denoted as $D_{1}$ and $D_{2}$, and the results of the nearness calculation are as follows: 


$$
\begin{aligned}
& N\left(D_{1}, E_{1}\right)=N\left(D_{1}^{(1)}, E_{1}\right)=1-\frac{1}{110}(0.773 \times 1+0.409 \times 2+0.364 \times 3)=0.976, \\
& N\left(D_{1}, E_{2}\right)=N\left(D_{1}^{(2)}, E_{1}\right)=1-\frac{1}{110}(0.591 \times 1+0.227 \times 2+0.364 \times 3)=0.981, \\
& N\left(D_{1}, E_{3}\right)=N\left(D_{1}^{(3)}, E_{1}\right)=1-\frac{1}{110}(0.636 \times 1+0.409 \times 2+0.227 \times 3)=0.981, \\
& N\left(D_{2}, E_{1}\right)=N\left(D_{2}^{(1)}, E_{1}\right)=1-\frac{1}{110}(0.507 \times 1+0.507 \times 2+0 \times 3)=0.986, \\
& N\left(D_{2}, E_{2}\right)=N\left(D_{2}^{(2)}, E_{1}\right)=1-\frac{1}{110}(0.493 \times 1+0.493 \times 2+0 \times 3)=0.987, \\
& N\left(D_{2}, E_{3}\right)=N\left(D_{2}^{(3)}, E_{1}\right)=1-\frac{1}{110}(1 \times 1+0.507 \times 2+0.493 \times 3)=0.967 .
\end{aligned}
$$

After processing the fuzzy evaluation results controlled by two different membership function forms using the nearness degree algorithm, it is found that when the factor set defined by the Gaussian-type membership function is considered the value of the membership degree in the comment set, the degree of the blasting effect evaluation which belongs to the common comment set is consistent with that which belongs to the poor comment set; that is, the final evaluation result of the blasting effect using the Gaussian-type membership function is between the "common" and "poor" grades. Similarly, the degree of the blasting effect evaluation that belongs to "better" is essentially consistent with that which belongs to "common" when using the triangle membership function, which demonstrates that the final evaluation result of the blasting effect using the triangle membership function is between the "better" and "common" grades.

Through further observation of the blasting site and postblasting effect, we found that the indices of explosive consumption and detonator consumption in the blasting construction process are slightly beyond the reasonable range; the longest cast distance and blasting output per meter hole are also slightly less than the reasonable values given in $[16,17,25]$, and there are obvious toe rocks and bulks; loose rock poured into goaf accounts for approximately $20 \%$ of the stripped rock mass, and the accumulation of the big pile is not ideal. Thus, the result of the subjective blasting effect evaluation should be consistent with the final result of the fuzzy comprehensive evaluation under the control of the Gaussian membership function after processing the nearness algorithm. The evaluation results controlled by two different membership functions can all be considered common by the nearness algorithm, which is because the number of comment sets selected is small (three comment sets are selected, which are better, common, and poor, in this paper). If the evaluation set in this paper extends to five or more items, then the results of the fuzzy comprehensive evaluation processed by the nearness algorithm, and the final evaluation results controlled by two different membership functions, will be different.

\section{Conclusions}

This paper proposes a new evaluation method for the nonlinear relationship between the blasting effect and evaluation factors. This method selects 11 evaluation indicators to evaluate the postexplosion effect. Part of the indicator parameters are derived from field test monitoring, and another part of the indicator parameters are derived from numerical simulation. The main conclusions of this study are as follows:

(1) Two different membership functions are defined for fuzzy evaluation of the blasting effect. Gaussian and triangle membership functions are selected, respectively. The preliminary evaluation results of the two membership functions are common. But the initial results have a large difference in the weight of the comment set, so the initial evaluation results are not credible. It is essential to further optimize the evaluation results by the proximity analysis method. The appraisal result is between common and poor with the Gaussian membership function, while the result is between better and common with the triangle membership function. Through the observation, it is found that the local blasting effect is not ideal, and the evaluation result of the Gaussian membership function is closer to the actual situation. Therefore, the Gaussian function instead of the linear function should be adopted to define the membership function of each index when nonlinear factors such as the blasting effect are selected to measure the evaluation results of the target layer.

(2) Slope blasting is the problem of fracture damage of the rock mass under blasting impact load. The rupture process includes two parts: the rupture effect of tensile cracking and compression hardening. Therefore, the establishment of a reasonable constitutive relationship of tensile and compression damage can accurately simulate the blasting phenomenon of the rock mass. It is assumed that the compression state of the rock mass under the impact load obeys Mises yield failure, the stress- 
strain relationship satisfies the bilinear elastoplastic constitutive relation, and then the constitutive damage model of hardening effect under compressive stress can be constructed. In contrast, the rock mass satisfies the TCK relationship under the impact load, the material response obeys the elastic Hooke's law, and the calculation model of the fracture damage can be constructed. By coupling the rock mass fracture damage calculation model and the compressive stress hardening effect constitutive relationship, the constitutive relation of rock mass tensile compression damage can be constructed. And the user-defined material interface function provided by LS-DYNA is used to embed the tensile and compressive damage evolution model into the elastoplastic constitutive material to accurately simulate the blasting effect of the slope.

(3) Based on the actual postexplosion situation and similar simulation tests, the 11 evaluation indicators in this paper are assigned. The strongest indicator of the impact on the blasting effect obtained through fuzzy calculation is boulder yield, and the weakest indicator is postcracking distance. The impact of each indicator varies greatly, and the weight of the strongest indicator is about 10 times that of the weakest. The 11 indicators are arranged in the order of impact from strong to weak as follows: boulder yield (23\%), explosive consumption (18.4\%), blasted volume per meter of hole $(15.7 \%)$, throw rate $(11.3 \%)$, vibration velocity $(9.2 \%)$, loose coefficient (6.8\%), cast distance $(4.7 \%)$, root rate $(4 \%)$, flying distance of flyrock (2.9\%), detonator unit consumption $(2.5 \%)$, and postcracking distance $(2.1 \%)$.

\section{Data Availability}

All data generated or analyzed during this study are included in this article.

\section{Conflicts of Interest}

The authors declare that they have no conflicts of interest.

\section{Acknowledgments}

This research was financially supported by the National Natural Science Foundation of China (51904138 and 51076061) and the Natural Science Foundation of Gansu Province, China (B061709).

\section{References}

[1] C. Kuzu, A. Fisne, and S. G. Ercelebi, "Operational and geological parameters in the assessing blast induced airblastoverpressure in quarries," Applied Acoustics, vol. 70, no. 3, pp. 404-411, 2009.

[2] S. P. Singh, "New trends in drilling and blasting technology," International Journal of Surface Mining, Reclamation and Environment, vol. 14, no. 4, pp. 305-315, 2000.
[3] Z.-w. Wang, X.-b. Li, K. Peng, and J.-f. Xie, "Impact of blasting parameters on vibration signal spectrum: determination and statistical evidence," Tunnelling and Underground Space Technology, vol. 48, pp. 94-100, 2015.

[4] J. Xu, Y. Kang, X. Wang, G. Feng, and Z. Wang, "Dynamic characteristics and safety criterion of deep rock mine opening under blast loading," International Journal of Rock Mechanics and Mining Science, vol. 119, pp. 156-167, 2018.

[5] P. Yan, W. Lu, J. Zhang, Y. Zou, and M. Chen, "Evaluation of human response to blasting vibration from excavation of a large scale rock slope: a case study," Earthquake Engineering and Engineering Vibration, vol. 16, no. 2, pp. 435-446, 2017.

[6] Y. K. Chen, J. H. Xu, X. H. Huo, and J. C. Wang, "Numerical simulation of dynamic damage and stability of a bedding rock slope under blasting load," Shock and Vibration, vol. 2019, Article ID 9616859, 13 pages, 2019.

[7] D. G. Albert, S. Taherzadeh, K. Attenborough, P. Boulanger, and S. N. Decato, "Ground vibrations produced by surface and near-surface explosions," Applied Acoustics, vol. 74, no. 11, pp. 1279-1296, 2013.

[8] L. An, F. T. Suorineni, S. Xu, Y.-H. Li, and Z.-C. Wang, "A feasibility study on confinement effect on blasting performance in narrow vein mining through numerical modelling," International Journal of Rock Mechanics and Mining Sciences, vol. 112, pp. 84-94, 2018.

[9] K. Liu and B. Liu, "Optimization of smooth blasting parameters for mountain tunnel construction with specified control indices based on a GA and ISVR coupling algorithm," Tunnelling and Underground Space Technology, vol. 70, pp. 363-374, 2017.

[10] M. Monjezi, H. Amini Khoshalan, and A. Yazdian Varjani, "Optimization of open pit blast parameters using genetic algorithm," International Journal of Rock Mechanics and Mining Sciences, vol. 48, no. 5, pp. 864-869, 2011.

[11] Y.-H. Jong and C.-I. Lee, "Influence of geological conditions on the powder factor for tunnel blasting," International Journal of Rock Mechanics and Mining Sciences, vol. 41, pp. 533-538, 2004.

[12] X. Xue, "Neuro-fuzzy based approach for prediction of blastinduced ground vibration," Applied Acoustics, vol. 152, pp. 73-78, 2019.

[13] X. Ding, K. Li, S. Xiao, and W. Hu, "Analysis of key technologies and development of integrated digital processing system for cast blasting design," Journal of Central South University, vol. 22, pp. 1027-1044, 2015.

[14] M. Dadashzadeh, R. Abbassi, F. Khan, and K. Hawboldt, "Explosion modeling and analysis of BP deepwater horizon accident," Safety Science, vol. 57, pp. 150-160, 2013.

[15] M. Monjezi, M. Rezaei, and A. Yazdian Varjani, "Prediction of rock fragmentation due to blasting in Gol-E-Gohar iron mine using fuzzy logic," International Journal of Rock Mechanics and Mining Sciences, vol. 46, no. 8, pp. 1273-1280, 2009.

[16] X. Li, S. Zhang, Y. Xu, and T. Hu, "Study on the bench castblasting effects influenced by explosive specific charge," Transactions of Beijing Institute of Technology, vol. 36, no. 12, pp. 1233-1236, 2016.

[17] Z. Lei, R. Yang, and T. Tao, "Comprehensive evaluation of bench blasting effect based on uncertainty measurement theory," Journal of China Coal Society, vol. 40, no. 2, pp. 353-359, 2015.

[18] Y. Li and Z. Shi, "Weak uninorm aggregation operators," Information Sciences, vol. 124, no. 1-4, pp. 317-323, 2000.

[19] W.-X. Li, D.-L. Qi, S.-F. Zheng, J.-C. Ren, J.-f. Li, and X. Yin, "Fuzzy mathematics model and its numerical method of 
stability analysis on rock slope of opencast metal mine," Applied Mathematical Modelling, vol. 39, no. 7, pp. 17841793, 2015.

[20] Y. Zhou, L. Lin, D. Wang, M. He, and D. He, "A new method to classify railway vehicle axle fatigue crack AE signal," Applied Acoustics, vol. 131, pp. 174-185, 2018.

[21] A. Maristany, M. R. López, and C. A. Rivera, "Soundscape quality analysis by fuzzy logic: a field study in Cordoba, Argentina," Applied Acoustics, vol. 111, pp. 106-115, 2016.

[22] Y. J. Zhang, Y. J. Li, S. J. Li, and Q. Jiang, "Study of boundaries of membership function values for slope fuzzy reliability analysis," Rock and Soil Mechanics, vol. 35, pp. 1157-1163, 2014.

[23] C. H. Juang, Y.-Y. Jhi, and D.-H. Lee, "Stability analysis of existing slopes considering uncertainty," Engineering Geology, vol. 49, no. 2, pp. 111-122, 1998.

[24] Z. Su, D. Hu, and X. C. Yu, "General interval approach for encoding words into interval type-2 fuzzy sets based on normal distribution and free parameter," Soft Computing, vol. 23, no. 17, pp. 8187-8206, 2018.

[25] W. B. Sun, X. L. Liu, H. B. Wang, and F. Liu, "Weight analysis of cast blasting effective factors based on MIV method," Journal of China University of Mining and Technology, vol. 41, no. 6, pp. 963-998, 2012.

[26] N. Jiang, C. Zhou, W. Ping, X. Xu, and S. Lu, "Altitude effect of blasting vibration velocity in rock slopes," Journal of Central South University (Science and Technology), vol. 45, no. 1, pp. 237-243, 2014.

[27] G. W. Ma and X. M. An, "Numerical simulation of blastinginduced rock fractures," International Journal of Rock Mechanics and Mining Sciences, vol. 45, no. 6, pp. 966-975, 2008.

[28] X. P. Li, J. L. Lv, J. H. Huang, Y. Luo, and T. T. Liu, "Numerical simulation research of smooth wall blasting using the timing sequence control method under different primary blast hole shapes," Shock and Vibration, vol. 2019, Article ID 2425904, 16 pages, 2019.

[29] H. Hu, W. B. Lu, P. Yan, M. Chen, and Q. Gao, “A vibrationisolating blast technique with shock-reflection device for dam foundation excavation in complicated geological conditions," Shock and Vibration, vol. 2018, Article ID 8029513, 11 pages, 2018.

[30] G. Suazo and G. Villavicencio, "Numerical simulation of the blast response of cemented paste backfilled stopes," Computers and Geotechnics, vol. 100, pp. 1-14, 2018.

[31] B. Budiansky and R. J. O'connell, "Elastic moduli of a cracked solid," International Journal of Solids and Structures, vol. 12, no. 2, pp. 81-97, 1976.

[32] L. X. Xie, W. B. Lu, Q. B. Zhang, Q. H. Jiang, G. H. Wang, and J. Zhao, "Damage evolution mechanisms of rock in deep tunnels induced by cut blasting," Tunnelling and Underground Space Technology, vol. 58, pp. 257-270, 2016.

[33] D. E. Grady and M. E. Kipp, "Continuum modelling of explosive fracture in oil shale," International Journal of Rock Mechanics and Mining Sciences \& Geomechanics Abstracts, vol. 17, no. 3, pp. 147-157, 1980.

[34] L. M. Taylor, E.-P. Chen, and J. S. Kuszmaul, "Microcrackinduced damage accumulation in brittle rock under dynamic loading," Computer Methods in Applied Mechanics and Engineering, vol. 55, no. 3, pp. 301-320, 1986.

[35] S. Zhang, W. Liu, and H. Lv, "Creep energy damage model of rock graded loading," Results in Physics, vol. 12, pp. 11191125, 2019.
[36] Y. Huang, D. Liu, S. Li, X. Li, and J. Wang, "Numerical simulation on pinpoint blasting of sloping surface," Explosion and Shock Waves, vol. 34, no. 4, pp. 495-500, 2014.

[37] Z. L. Zhou, R. S. Cheng, X. Cai, J. L. Jia, and W. H. Wang, "Comparison of presplit and smooth blasting methods for excavation of rock wells," Shock and Vibration, vol. 2019, Article ID 3743028, 12 pages, 2019. 